\title{
Control-Oriented Linear Parameter-Varying Modelling of a Turbocharged Diesel Engine
}

\author{
Merten Jung \\ University of Cambridge \\ Trumpington Street \\ Cambridge, CB2 1PZ, UK \\ Email: Jung@tu-harburg.de
}

\author{
Keith Glover \\ University of Cambridge \\ Trumpington Street \\ Cambridge, CB2 1PZ, UK \\ Email: kg@eng.cam.ac.uk
}

\begin{abstract}
In this paper, a third order nonlinear model of the airpath of a turbocharged diesel engine is derived, which is then converted into linear parameter-varying (LPV) form with the intake and exhaust manifold pressure as parameters. The model predicts the mass air flow (MAF) and manifold absolute pressure (MAP) based on the EGR and VGT actuator positions, speed, and load. A comparison to experimental data from the engine test bed and to a higher order nonlinear model suggests the validity of this approach.
\end{abstract}

\section{INTRODUCTION}

Modern diesel engines are typically equipped with variable geometry turbochargers (VGT) and exhaust gas recirculation (EGR), which both introduce feedback loops from the exhaust to the intake manifold. This leads to a substantial increase in calibration effort. Model-based control aims at reducing this effort, but the controllers have to be robust for successful implementation on the engine. Typically, several controllers are designed for different speed-load points and are then merged in a gain scheduling approach, which does not give robustness guarantees anymore [1]. This problem can be overcome by recently developed robust gain scheduling methodologies (see e. g. [2]) that require a linear parameter-varying model of the plant, which is usually difficult to obtain.

LPV systems, which where initially studied in [3], are linear systems whose describing matrices depend on an exogenous time-varying parameter vector $\rho(t)$ :

$$
\begin{aligned}
\dot{x} & =A(\rho(t)) x+B(\rho(t)) u \\
y & =C(\rho(t)) x+D(\rho(t)) u
\end{aligned}
$$

where $A, B, C, D$ are continuous matrix valued functions of $\rho(t)$, which varies in the set of continuously differentiable parameter curves $\rho:[0, \infty) \rightarrow \mathbb{R}^{k}$. Both $\rho(t)$ and its rate of variation $\dot{\rho}$ are contained in prespecified compact sets $\Gamma$ and $\Gamma_{d}$. The parameter vector $\rho$ is composed of different real parameters $\rho_{i}$ each one of them varying between $\underline{\rho}_{i}$ and $\bar{\rho}_{i}$ :

$$
\rho_{i}(t) \in\left[\underline{\rho}_{i}, \bar{\rho}_{i}\right], \forall t \geq 0, \quad i=1, \ldots, k .
$$

The rate of variation $\dot{\rho}_{i}$ is assumed to be well-defined at all times and satisfies

$$
\dot{\rho}_{i}(t) \in\left[\underline{q}_{i}, \bar{q}_{i}\right], \quad \forall t \geq 0, \quad i=1, \ldots, k .
$$

The exogenous parameter $\rho(t)$ is unknown apriori but can be measured or estimated online. This distinguishes LPV systems from linear time-varying systems for which the timevariations are known beforehand. If the scheduling parameter is endogenous to the state dynamics, e. g. $\rho(t)$ is a state itself as it will be the case for the airpath model, the system shall be called quasi-LPV [4].

Note that the parameters are allowed to enter the system matrices in a nonlinear way. Nevertheless, it is typically rather difficult to convert a complex nonlinear model into (quasi) LPV form, although many nonlinear systems of interest can be written in that form. A common problem is that the dynamics of the plant are not linear in the plant input. Another issue is that the order of the system should be as small as possible because the control design and implementation are computationally expensive.

After introducing the control problem in Section II, a simplified nonlinear airpath model of the diesel engine will be described in Section III. The model will be derived and parameterised for low and medium speed-load points, which are covered by the New European Drive Cycle (NEDC). It will then be converted into quasi-LPV form in Section IV. Finally, Section $\mathrm{V}$ will give some model validation results and a comparison to a full nonlinear model.

\section{The Control Problem}

The plant to be controlled is a turbocharged passenger car diesel engine equipped with exhaust gas recirculation (EGR) as depicted in Figure 1. The turbocharger increases the power density of the engine by forcing air into the cylinders, which allows injection of additional fuel without reaching the smoke limit. The turbine, which is driven by the energy in the exhaust gas, has a variable geometry (VGT) that allows the adaptation of the turbine efficiency based on the engine operating point.

The second feedback path from the exhaust to the intake manifold is due to exhaust gas recirculation, which is controlled by an EGR valve. The recirculated exhaust gases replace oxygen in the inlet charge, thereby reducing the temperature profile of the combustion and hence the emissions of oxides of nitrogen. The interactions are relatively complex; a detailed description can be found in [5] and the references therein. 


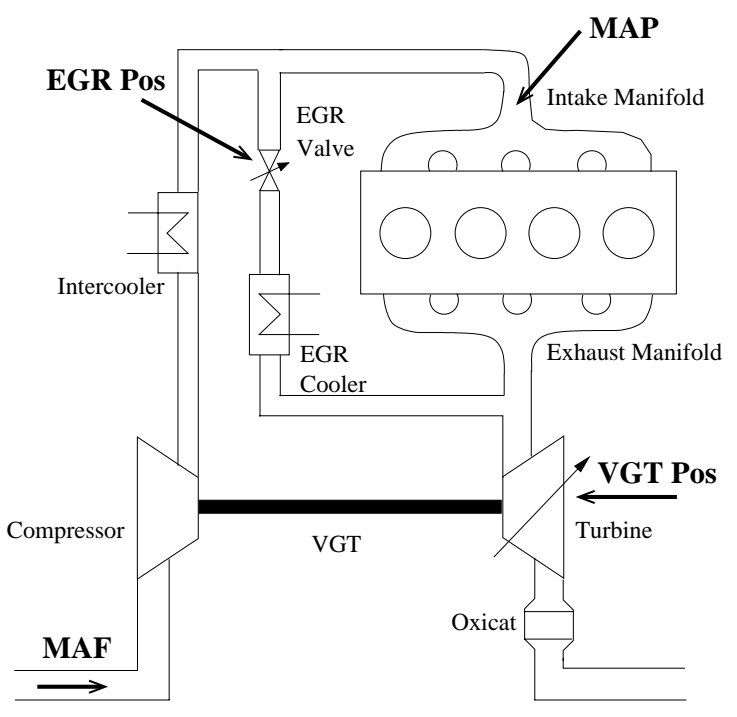

Fig. 1. Diesel engine setup.

While the VGT actuator is typically used to control the intake manifold absolute pressure (MAP), the EGR valve controls the mass air flow (MAF) into the engine. Both the EGR and VGT paths are driven by the exhaust gases and hence constitute an inherently multivariable control problem.

\section{Simplified Nonlinear Model}

For the detailed derivation of an eighth-order nonlinear mean-value model of the engine under investigation, the reader is referred to [6]. The model therein will serve as a benchmark for comparison of the quasi-LPV model developed here and is subsequently named the full nonlinear model.

In [7], Jankovic et al. propose a simplified third order nonlinear model in which the manifold dynamics are described solely by differentiating the ideal gas law $p V=R T m$ resulting in one differential equation for each the intake and the exhaust manifold pressure. The turbocharger dynamics are approximated by the power transfer with time constant $\tau$ :

$$
\begin{aligned}
\dot{p}_{i} & =\frac{R T_{i}}{V_{i}}\left(W_{c i}+W_{x i}-W_{i e}\right)+\frac{\dot{T}_{i}}{T_{i}} p_{i} \\
\dot{p}_{x} & =\frac{R T_{x}}{V_{x}}\left(W_{i e}+W_{f}-W_{x i}-W_{x t}\right)+\frac{\dot{T}_{x}}{T_{x}} p_{x} \\
\dot{P}_{c} & =\frac{1}{\tau}\left(-P_{c}+\eta_{m} P_{t}\right)
\end{aligned}
$$

Subsequently, the mechanical efficiency of the turbocharger $\eta_{m}$ will be set to unity. In a further approximation, the intake and exhaust manifold temperatures are assumed to be constant such that the effect of $\dot{T}_{i}$ and $\dot{T}_{x}$ on $p_{i}$ and $p_{x}$, respectively, is neglected. Alternatively, the derivatives could be neglected, but the measured temperatures could be kept as measured parameters (quasi-static approach) to improve the model. However, with LPV control design in mind, the number of parameters should be as small as possible, hence the choice to set these temperatures to constants. Since the
quasi-LPV model will be used for a whole range of speeds and loads (i.e. those covered on the NEDC), this assumption limits the accuracy of the model and the constant temperatures will be optimised to give a good representation of the airpath dynamics over the NEDC as will be discussed below.

The precompressor and postturbine pressures are fixed at ambient conditions. The relation between compressor flow and power is given by

$$
W_{c i}=\frac{\eta_{c}}{c_{p} T_{a}} \frac{P_{c}}{\left(\frac{p_{i}}{p_{a}}\right)^{\mu}-1} .
$$

Note that $\mu:=\frac{\gamma-1}{\gamma}=0.286$. The compressor efficiency is assumed to be constant rather than being parameterised as a function of turbocharger speed and pressure ratio across the compressor as in the full nonlinear model.

The flow through the EGR valve is approximated for subsonic conditions as suggested in [8] by

$$
W_{x i}=\frac{A_{r}\left(x_{r}\right) p_{x}}{\sqrt{R T_{x}}} \sqrt{2 \frac{p_{i}}{p_{x}}\left(1-\frac{p_{i}}{p_{x}}\right)},
$$

where the effective area of the EGR valve $A_{r}$ is a quadratic polynomial in the valve lift $x_{r}$. In order to avoid the input $x_{r}$ entering the equations quadratically, the static nonlinearity from actuator position to effective area is pulled out of the model and the effective area $A_{r}$ is used as input directly. Figure 2 shows that this static nonlinearity is monotonically increasing and can therefore be inverted such that controllers can be designed with the effective area as output.

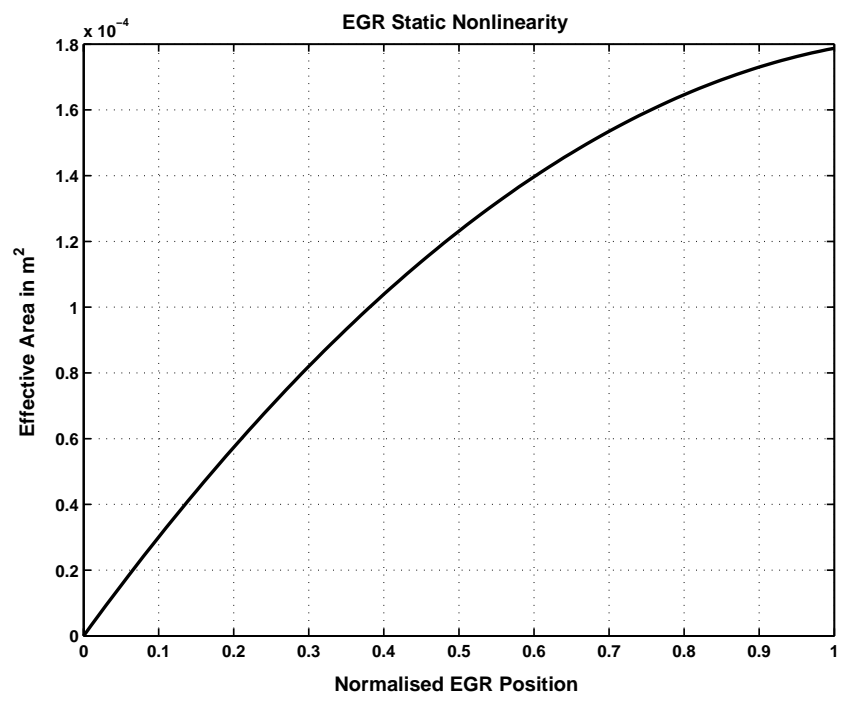

Fig. 2. Static nonlinearity of the EGR valve.

The mass flow rate from the intake manifold into the cylinders is determined by the speed-density equation:

$$
W_{i e}=\eta_{v} \frac{p_{i}}{T_{i} R} \frac{N}{60} \frac{V_{d}}{2}
$$

with the total displacement volume $V_{d}$ and the volumetric efficiency $\eta_{v}$ assumed to be constant for this simplified model. 
The turbine flow $W_{x t}$ is parameterised based on the standard orifice flow equation. The effective area is identified as a linear function of the VGT position. Applying the same approximation for the flow function as in (4) the following parameterisation with identified parameters $a, b, c, d$ is obtained:

$$
\begin{aligned}
W_{x t} & =\left(a x_{v}+b\right)\left(c\left(\frac{p_{x}}{p_{a}}-1\right)+d\right) \frac{p_{x}}{p_{r e f}} \\
& \times \sqrt{\frac{T_{r e f}}{T_{x}}} \sqrt{2 \frac{p_{a}}{p_{x}}\left(1-\frac{p_{a}}{p_{x}}\right)}, \\
a & =490.4, b=633.7, c=0.4, d=0.6,
\end{aligned}
$$

where the reference temperature and pressure are chosen to $298 \mathrm{~K}, 101.3 \mathrm{kPa}$. Figure 3 shows the fit (solid) compared to experimental (dot) and provided (dash) data. Note that the fit was chosen to match the experimental data especially at the low pressure ratios which are encountered on the NEDC.

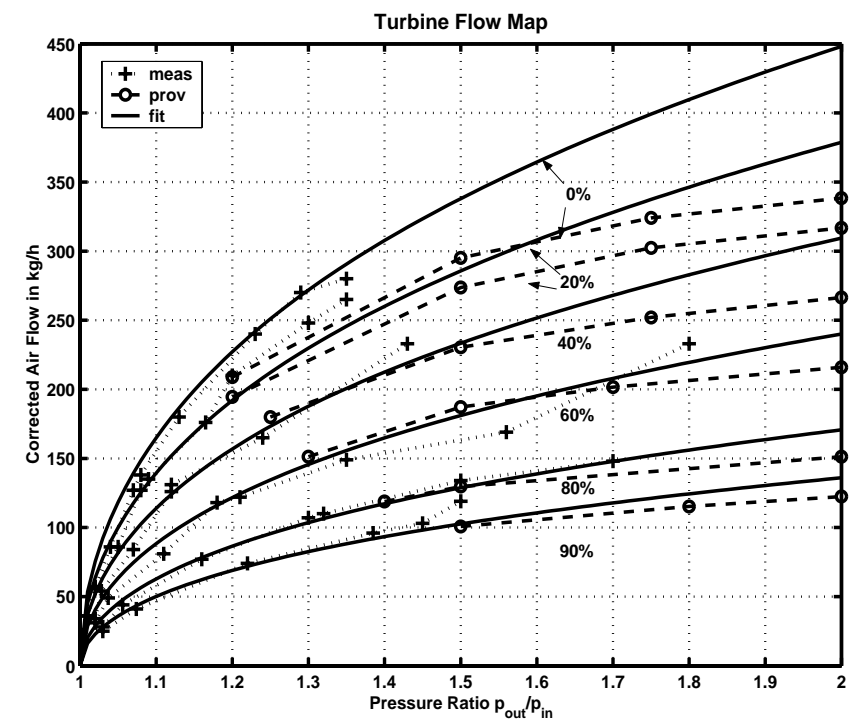

Fig. 3. Turbine flow map for the quasi-LPV model: Measured (dot), provided (dash), and fitted (solid) data for different VGT positions.

Finally, the turbine power is given as (note that the turbine efficiency is also set to a constant in this simplified model):

$$
P_{t}=W_{x t} c_{p} T_{x} \eta_{t}\left(1-\left(\frac{p_{a}}{p_{x}}\right)^{\mu}\right) .
$$

As opposed to the full nonlinear model, the simplified version contains several constant parameters, namely the compressor and turbine efficiencies $\eta_{c}$ and $\eta_{t}$, the volumetric efficiency $\eta_{v}$, the intake and exhaust manifold temperatures $T_{i}$ and $T_{x}$, and the time constant of the turbocharger power transfer $\tau$. Obviously, these parameters vary with engine conditions and keeping them constant is only a crude approximation. However, it turns out that they capture the dynamics of the system at least in the low and medium speed-load region, which is under investigation here.

The aforementioned parameters are chosen based on a nonlinear optimisation. Therefore, the model is simulated with
TABLE I

PARAMETER VALUES OF THE SIMPLIFIED MODEL.

\begin{tabular}{|c|c|c|c|c|c|}
\hline$\eta_{c}$ in $\%$ & $\eta_{t}$ in $\%$ & $\eta_{v}$ in $\%$ & $\tau$ in $\mathrm{s}$ & $T_{i}$ in $\mathrm{K}$ & $T_{x}$ in $\mathrm{K}$ \\
\hline 61 & 76 & 87 & 0.11 & 313 & 509 \\
\hline
\end{tabular}

the inputs taken from extra-urban part of the NEDC, which covers the range of interest in this application. Matlab is then used to find the parameters which result in the best fit of the simulation and experimental data. As the optimisation is based on a nonlinear search, convergence to the global minimum cannot be guaranteed. However, the obtained parameter values, which are given in Table I, appear to be reasonable.

Note that the diesel engine model has a singularity at $p_{i}=p_{a}$ when the compressor flow (3) becomes infinite. Fortunately, it can be shown that the set $\Omega:=\left\{\left(p_{i}, p_{x}, P_{c}\right)\right.$ : $\left.p_{i}>p_{a}, p_{x}>p_{a}, P_{c}>0\right\}$ is invariant, i. e. every trajectory starting in $\Omega$ stays in $\Omega$ for all time [7].

\section{CONVERSION TO AN QUASI-LPV MODEL}

Combining and rearranging the equations from the previous section, the following three nonlinear differential equations are obtained:

$$
\begin{aligned}
& \dot{p}_{i}=\frac{R T_{i}}{V_{i}} \frac{\eta_{c}}{c_{p} T_{a}} \frac{\mathbf{P}_{\mathbf{c}}}{\left(\frac{p_{i}}{p_{a}}\right)^{\mu}-1}-\frac{p_{i}}{V_{i}} \frac{\eta_{v} V_{d}}{2 \cdot 60} \mathbf{N} \\
& +\frac{R T_{i}}{V_{i}} \frac{p_{x}}{\sqrt{R T_{x}}} \sqrt{2 \frac{p_{i}}{p_{x}}\left(1-\frac{p_{i}}{p_{x}}\right)} \mathbf{A}_{\mathbf{r}} \\
& \dot{p}_{x}=\frac{T_{x}}{T_{i}} \frac{p_{i}}{V_{x}} \frac{\eta_{v} V_{d}}{2 \cdot 60} \mathbf{N}-\frac{R T_{x}}{V_{x}} \frac{p_{x}}{\sqrt{R T_{x}}} \sqrt{2 \frac{p_{i}}{p_{x}}\left(1-\frac{p_{i}}{p_{x}}\right)} \mathbf{A}_{\mathbf{r}} \\
& -\frac{R T_{x}}{V_{x}} a\left(c\left(\frac{p_{x}}{p_{a}}-1\right)+d\right) \frac{p_{x}}{p_{r e f}} \\
& \times \sqrt{2 \frac{p_{a}}{p_{x}}\left(1-\frac{p_{a}}{p_{x}}\right)} \sqrt{\frac{T_{r e f}}{T_{x}}} \mathbf{x}_{\mathbf{v}} \\
& -\frac{R T_{x}}{V_{x}} b\left(c\left(\frac{p_{x}}{p_{a}}-1\right)+d\right) \frac{\mathbf{p}_{\mathbf{x}}}{p_{r e f}} \\
& \times \sqrt{2 \frac{p_{a}}{p_{x}}\left(1-\frac{p_{a}}{p_{x}}\right)} \sqrt{\frac{T_{r e f}}{T_{x}}} \\
& +\frac{R T_{x}}{V_{x}} \mathbf{W}_{\mathbf{f}} \\
& \dot{P}_{c}=-\frac{\mathbf{P}_{\mathbf{c}}}{\tau}+\frac{\eta_{t} c_{p} T_{x}}{\tau}\left(1-\left(\frac{p_{a}}{p_{x}}\right)^{\mu}\right) \frac{p_{x}}{p_{r e f}} \sqrt{\frac{T_{r e f}}{T_{x}}} \\
& \times a\left(c\left(\frac{p_{x}}{p_{a}}-1\right)+d\right) \sqrt{2 \frac{p_{a}}{p_{x}}\left(1-\frac{p_{a}}{p_{x}}\right)} \mathbf{x}_{\mathbf{v}} \\
& +\frac{\eta_{t} c_{p} T_{x}}{\tau}\left(1-\left(\frac{p_{a}}{p_{x}}\right)^{\mu}\right) \frac{\mathbf{p}_{\mathbf{x}}}{p_{\text {ref }}} \sqrt{\frac{T_{\text {ref }}}{T_{x}}} \\
& \times b\left(c\left(\frac{p_{x}}{p_{a}}-1\right)+d\right) \sqrt{2 \frac{p_{a}}{p_{x}}\left(1-\frac{p_{a}}{p_{x}}\right)}
\end{aligned}
$$



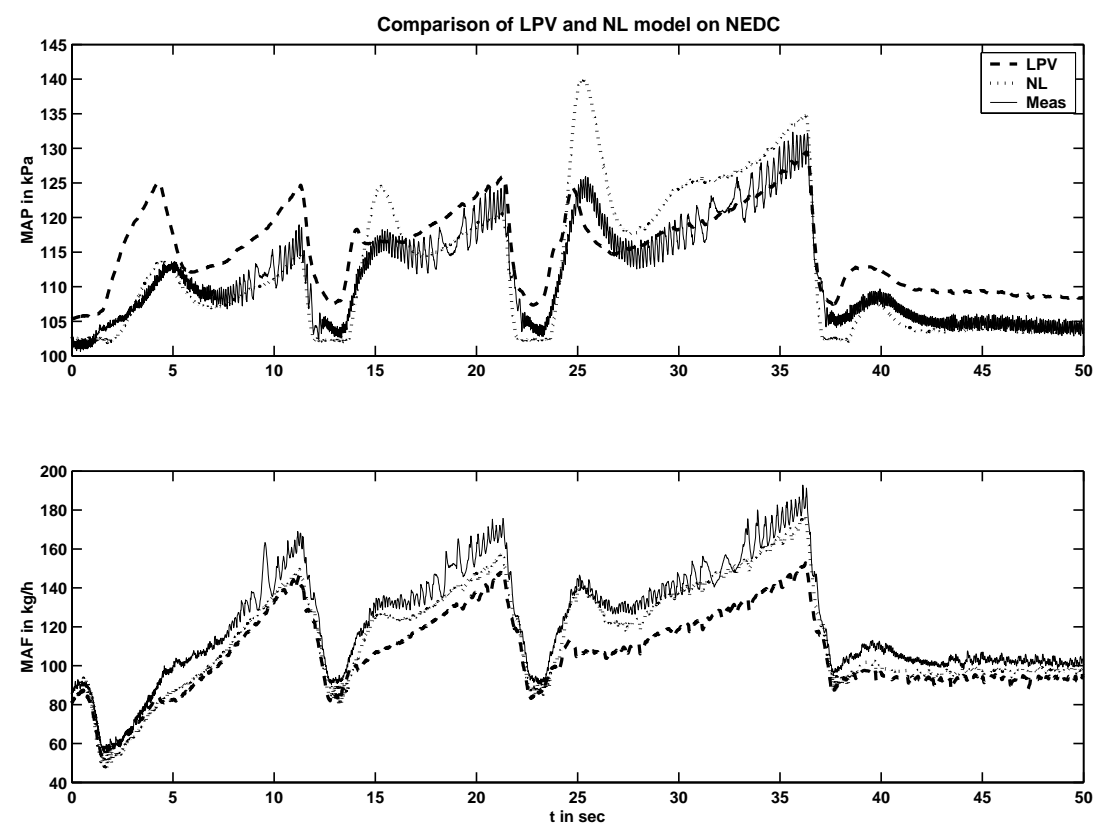

Fig. 4. Validation results for the quasi-LPV model (dash) on the NEDC in comparison to the experimental data (dot) and the simulation results for the full nonlinear model (solid).

These equations now have to be cast into the form (1). With the chosen states and inputs, the state equation becomes:

$$
\left(\begin{array}{c}
\dot{p}_{i} \\
\dot{p}_{x} \\
\dot{P}_{c}
\end{array}\right)=A(\rho(t))\left(\begin{array}{c}
p_{i} \\
p_{x} \\
P_{c}
\end{array}\right)+B(\rho(t))\left(\begin{array}{c}
A_{r} \\
x_{v} \\
N \\
W_{f}
\end{array}\right) .
$$

An inspection of (8) reveals that only the intake and exhaust manifold pressure $p_{i}$ and $p_{x}$ enter the equations in various nonlinear ways. Therefore, they have to be considered as parameters, i.e. $\rho_{1}=p_{i}$ and $\rho_{2}=p_{x}$. With that in mind, each term in (8) contains a state or input variable plotted in boldface. The terms multiplying these variables now only contain fixed parameters or the time-varying parameters $\rho_{1}$ and $\rho_{2}$. Note that in some terms $p_{x}$ is printed in boldface which means that there are no other states or inputs multiplying it and hence it can be left as a state.

In order to convert (8) to (9), all nonboldface printed $p_{i}$ and $p_{x}$ are replaced by $\rho_{1}$ and $\rho_{2}$, respectively, the terms multiplying states and inputs are separated, and everything is written as a matrix equation. In (9), the origin is an equilibrium point, but it does not correspond to a physical equilibrium point which will have intake and exhaust manifold pressures larger than $100 \mathrm{kPa}$. However, for the control design, the parameters $\rho$ are considered to be independent of the state $x$, i. e. the parameter space will be gridded. Assuming there exist equilibria $x_{e q}$ and $u_{e q}$ for each parameter value, (9) can be redefined by subtracting the equilibria from the states and inputs. However, this does not affect the $\mathrm{A}$ and $\mathrm{B}$ matrices. If the input to the controller is the error between reference and measured values (i. e., operating point offsets disappear in the controller input) and the controller will have integrating behaviour (such that the operating point offsets in $u$ can be provided by the integrators), a transformation of (9) to a physical equilibrium is not necessary. Otherwise, Shamma's state transformation can be applied to sort out the equilibrium conditions [4].

The significance of $p_{i}$ not appearing in boldface in (8) is that the first column of the $A$ matrix will be all zeros resulting in the system containing an integrator. This can be avoided if the speed input $N$ is redefined, e.g. $N=\tilde{N}+1500 \mathrm{rpm}$. Thus, the term multiplying $N$ in the first row can be restated as:

$$
-\frac{p_{i}}{V_{i}} \frac{\eta_{v} V_{d}}{2 \cdot 60} \mathbf{N}=-\frac{p_{i}}{V_{i}} \frac{\eta_{v} V_{d}}{2 \cdot 60} \tilde{\mathbf{N}}-\frac{\mathbf{p}_{\mathbf{i}}}{V_{i}} \frac{\eta_{v} V_{d}}{2 \cdot 60} 1500 .
$$

Now, the $(1,1)$ entry in the $A$ matrix becomes nonzero. The same applies to the second line in (8). In a further step, the EGR and VGT inputs are redefined as

$$
\begin{aligned}
A_{r} & =\tilde{A}_{r}+8.9 \cdot 10^{-5} \mathrm{~m}^{2} \\
x_{v} & =\tilde{x}_{v}+0.5,
\end{aligned}
$$

where the offsets correspond to the centre of the actuator range. Note that such a redefinition of the fuelling input $W_{f}$ would result in a constant term not multiplying any other state or input; it is hence left unchanged. Since the engine dynamics are not included in the model, zero fuel flow does not imply engine stalling; the fuel flow is only included because it contributes to the flow from the cylinders into the exhaust. Moreover, on the normal operating range of the diesel engine, the fuel flow is 18 to 70 times smaller than the air flow. Hence, assuming a nontypical zero fuel flow is not problematic.

The more general case, in which (8) contains terms, i. e. constants or even nonlinear functions in the scheduling parame- 
ters, that do not multiply any state or input can be converted into the form (9) using Shamma's state transformation [4].

The output equation in (1) can easily be obtained as

$$
\left(\begin{array}{c}
W_{c i} \\
p_{i}
\end{array}\right)=\left(\begin{array}{ccc}
0 & 0 & \frac{\eta_{c}}{c_{p} T_{a}} \frac{1}{\left(\frac{\rho_{1}}{p_{a}}\right)^{\mu}-1} \\
1 & 0 & 0
\end{array}\right)\left(\begin{array}{c}
p_{i} \\
p_{x} \\
P_{c}
\end{array}\right)
$$

where $p_{i}$ is replaced by $\rho_{1}$ and the $D$ matrix is entirely zero. All tildes will be omitted subsequently.

Note that $A_{r}$ and $x_{v}$ are the manipulated variables for this airpath submodel, while $N$ and $W_{f}$ are external disturbances which can be measured but not be manipulated. Hence, $N$ and $W_{f}$ could be introduced as additional parameters. For example, with $N$ as parameter, $p_{i}$ could be used as a state (described by a differential equation) rather than as an external parameter (which, by definition, can vary independently of the state $p_{i}$ ) in two more places. However, in order to keep the computational power required for LPV control design small, $N$ and $W_{f}$ are treated as external disturbances.

The non-uniqueness of the LPV representation of the nonlinear equations in (8) can make the results conservative. This issue of the state-dependent representation can be addressed by including an additional degree of freedom in the optimisation [9]. Here, the chosen representation yielded satisfactory performance in a subsequent control design such that its optimisation was not necessary.

Finally, note that there is no approximation involved when going from the simplified nonlinear model to the quasiLPV model. The validation in the following section therefore applies to both the simplified nonlinear and the quasi-LPV model.

\section{Model VAlidation}

In order to assess the accuracy of the simplified nonlinear model, it will be compared to the full nonlinear model and experimental results with a focus on MAF $\left(W_{c i}\right)$ and MAP $\left(p_{i}\right)$ predictions. Figure 4 shows the simulation results for the part of the NEDC.

The comparison to the full nonlinear model shows that the steady-state offset is larger in both MAF and MAP for the quasi-LPV model, especially during the first ten seconds, but the latter captures the transient behaviour rather well considering the crude approximations involved in the derivation. Interestingly, during the transient between 25 and 35 seconds, MAP is better matched than by the full nonlinear model, which shows a large overshoot. However, this occurs at the expense of an underestimation of MAF. On the contrary, MAF is well matched during the first ten seconds, where MAP is overestimated by the quasi-LPV model. It should be pointed out that steady-state offsets are not of concern since any reasonably designed controller will take care of that.

Note that the simulation is entirely open loop and that speed and load vary significantly (750-2000 rpm, $-35-140 \mathrm{Nm})$. The EGR valve is kept shut for this experiment to separate the effect of the EGR path from the VGT path. The parameter estimation for the quasi-LPV model was obtained from the complete extra-urban part of the NEDC (i.e. $400 \mathrm{~s}$ ) with actuated EGR valve.

The further validation will focus on a fixed operating, namely $1500 \mathrm{rpm}, 85 \mathrm{Nm}$. Figure 5 shows the response of the quasi-LPV model to step inputs in EGR and VGT. On the engine, a pneumatic actuator (so-called EVRV) converts a duty cycle command to a vacuum pressure which results in an actuator position. The top plots in Figure 5 show the duty cycle as well as the measured actuator position which is then fed into the model.

The gains in the main couplings are not as well matched as for the full nonlinear model. While the gain in the EGR to MAF channel is slightly underestimated, it is overestimated for the VGT to MAP channel. This is not surprising when comparing the parameterisation effort that went into the full nonlinear model.

Concerning the cross-couplings, the gain in the EGR to MAP channel is actually better matched for the quasi-LPV model than for the nonlinear model. Moreover, the nonminimum phase behaviour of this channel is also reflected in the quasi-LPV model although it is hard to see in the figure. The cross-coupling from VGT to MAF shows some transient response but the steady-state is almost unchanged. This can be explained by the sensitivity of that gain to the effective area of the EGR valve, where the gain even undergoes a sign change for a sweep in the EGR position.

\section{CONCLUSION}

This paper has described linear parameter-varying modelling of the airpath of a turbocharged diesel engine. A third order nonlinear model is derived and directly converted to LPV form which requires that two states of the system (intake and exhaust manifold pressure) are considered as scheduling variables (quasi-LPV). The inputs to the model are EGR and VGT actuator positions, speed, and load. The outputs are chosen as intake manifold absolute pressure (MAP) and manifold air flow (MAF). Some rather crude approximations, e. g. assuming constant temperatures in the intake and exhaust manifold, facilitate the generation of a nonlinear model that allows direct conversion to LPV form. Nonlinear models, which do not allow such a conversion, can typically be approximated by quasi-LPV models. However, to find suitable approximations is a substantial task.

Despite the approximations, a comparison to simulation data from a higher order nonlinear model and to experimental data shows that the quasi-LPV model captures the nonlinearities and dynamics rather well. In the mean-time, a successful LPV control design based on the model described in this paper has been achieved; the results will be published in near future.

\section{ACKNOWLEDGMENT}

The principal author acknowledges financial support in part by the European Commission through the program Training and Mobility of Researchers - Research Networks and through project System Identification (FMRX CT98 0206) and acknowledges contacts with the participants in the European 

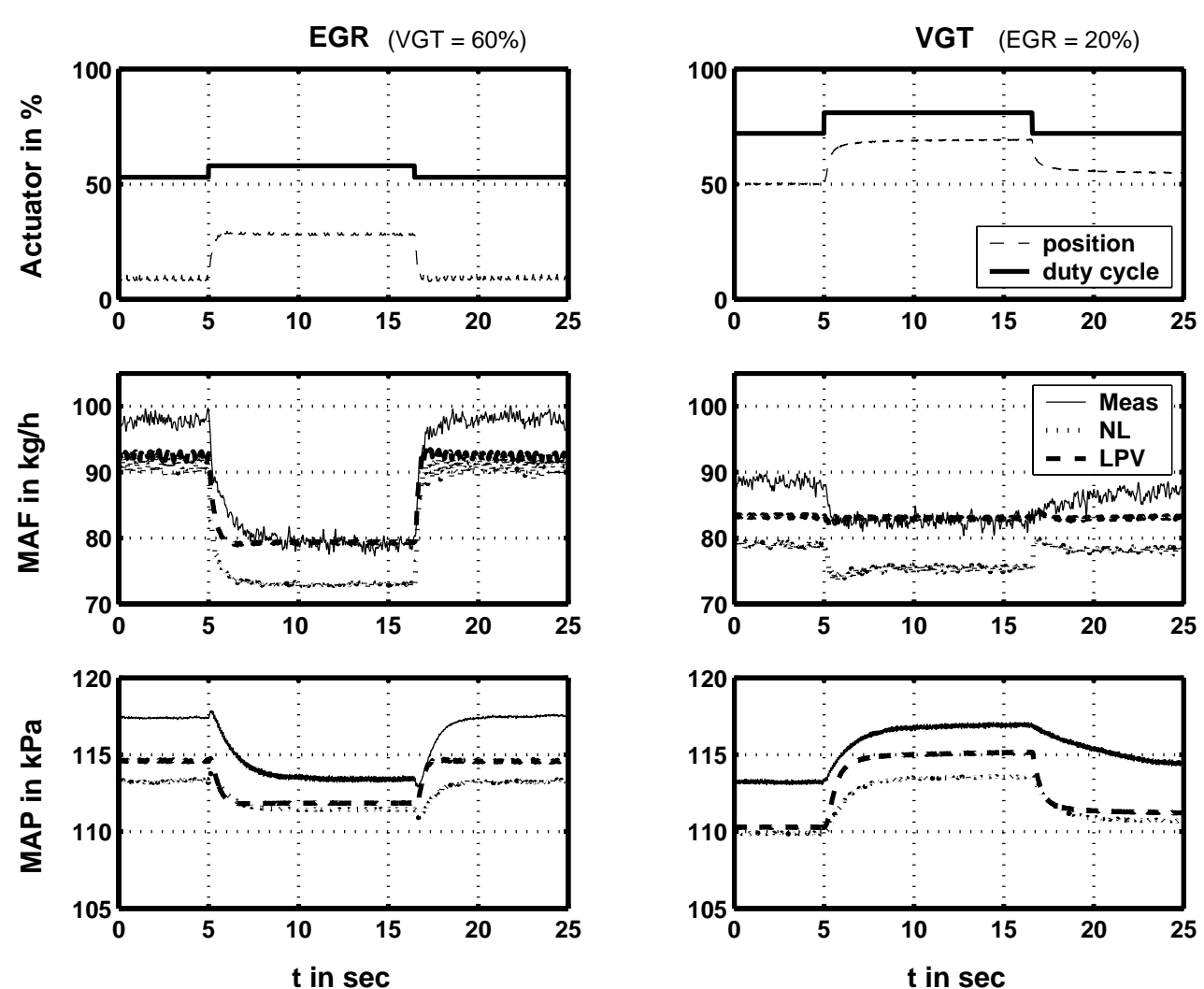

Fig. 5. Comparison of the MAF and MAP response to step inputs in EGR and VGT; quasi-LPV model (dash), experimental data (solid), nonlinear model (dot).

Research Network System Identification (ERNSI). Additional financial and technical support from the Ford Motor Company is also gratefully acknowledged.

\section{REFERENCES}

[1] J. S. Shamma, M. Athans. Gain Scheduling: Potential Hazards and Possible Remedies, IEEE Control Systems Magazine, Vol. 12, No. 3, pp. 101-107, 1991.

[2] P. Apkarian, R. J. Adams. Advanced Gain-Scheduling Techniques for Uncertain Systems, IEEE Transactions on Control Systems Technology, Vol. 6, No. 1, pp. 21-32, 1998.

[3] J. S. Shamma, M. Athans. Guaranteed Properties of Gain Scheduled Control for Linear Parameter-Varying Plants, Automatica, Vol. 27, No. 3 , pp. 559-564, 1991.

[4] J. S. Shamma, J. R. Cloutier. Gain-Scheduled Missile Autopilot Design Using Linear Parameter-Varying Transformations, Journal of Guidance, Control, and Dynamics, Vol. 16, No. 2, pp. 256-263, 1993.

[5] N. Ladommatos, S. Abdelhalim, H. Zhao, Z. Hu. The Dilution, Chemical and Thermal Effects of Exhaust Gas Recirculation on Diesel Engine Emissions - Part 1: Effect of Reducing Inlet Charge Oxygen, SAE, Paper 961165, 1996.

[6] M. Jung, R. Ford, K. Glover, N. Collings, U. Christen, M. Watts. Parameterization and Transient Validation of a Variable Geometry Turbocharger for Mean-Value Modeling at Low and Medium Speed-Load Points, Society of Automotive Engineers, Paper 2002-01-2729, 2002.

[7] M. Jankovic, M. Jankovic, I. Kolmanovsky. Robust Nonlinear Controller for Turbocharged Diesel Engines, Proceedings of the American Control Conference, Philadelphia, PA, 1998.

[8] L. Guzzella, A. Amstutz. Control of Diesel Engines, IEEE Control Systems Magazine, Vol. 18, No. 5, pp. 53-71, 1998

[9] Y. Huang, A. Jadbabaie. Nonlinear $\mathcal{H}_{\infty}$ Control: An Enhanced QuasiLPV Approach, Proceedings of the IFAC World Congress, Beijing, China, 1999.

\section{NOTATION}

Subscripts:

a ambient

c compressor

e engine (cylinders)

i intake manifold

$r$ recirculation $(\mathrm{EGR})$

$\mathrm{t}$ turbine

$\mathrm{x}$ exhaust manifold

Mass flows are denoted with two subscripts, indicating the source and the sink. For instance, $W_{c i}$ is the flow from the compressor into the intake manifold, i. e. MAF.

$A_{r} \quad \mathrm{~m}^{2} \quad$ effective area of EGR valve

$c_{p} \quad \mathrm{~J} / \mathrm{kg} / \mathrm{K} \quad$ specific heat ratio at constant pressure

$N \quad$ rpm engine speed

$P \quad$ W $\quad$ power

$p \quad \mathrm{kPa} \quad$ pressure

$R \quad \mathrm{~J} / \mathrm{kg} / \mathrm{K} \quad$ gas constant

$T \quad \mathrm{~K}$ temperature

$V \quad \mathrm{~m}^{3} \quad$ volume

$W_{a b} \quad \mathrm{~kg} / \mathrm{h} \quad$ mass flow from a to $\mathrm{b}$

$\gamma \quad$ - $\quad$ specific heat ratio $\left(\gamma=c_{p} / c_{v}\right)$

$\eta \quad$ - $\quad$ efficiency

$\tau \quad$ s $\quad$ time constant 